Pacific Journal of Mathematics

POINTWISE BOUNDS FOR THE SECOND INITIAL-BOUNDARY
VALUE PROBLEM OF PARABOLIC TYPE 


\title{
POINTWISE BOUNDS FOR THE SECOND INITIAL-BOUNDARY VALUE PROBLEM OF PARABOLIC TYPE
}

\author{
F. J. BellaR, JR.
}

By means of the divergence theorem and certain wellknown inequalities, the author presents explicit a priori pointwise bounds for the solution of the linear and nonlinear second initial-boundary value problem of the parabolic type. The desired result is obtained by using the parabolic form of Green's second identity with an appropriately defined parametrix serving as the first function of the identity and the difference of the solution and an arbitrary function which approximates the given data as the second. By means of various well-known inequalities, the unknown integrals in the resulting expression are bounded in terms of volume and surface integrals of the square of known functions. In the linear case the form of the bound is such that it may be improved by employing the Rayleigh-Ritz technique.

Background. In [8], Payne and Weinberger presented a method for determining similar bounds for the solution of Dirichlet and mixed type boundary value problems for certain second order elliptic equations and rather general domains. The results were extended by Bramble and Payne [2] and by the author [1] to the Neumann Problem for second order uniformly elliptic operators. In sequels the author will consider the first initial-boundary value problem of the parabolic type, degenerate parabolic problems of various types and nonlinear elliptic problems.

The results presented herein are applicable to problems for which existence and uniqueness theorems have not as yet been established. Assuming the existence of a solution, its uniqueness follows immediately from the form of the pointwise bound.

Since bounds for various quadratic functionals are also obtained in terms of the given data, it is assumed that the results of this paper may profitably be employed in the derivation of such existence proofs. For information pertaining to parabolic problems for which solutions are known to exist, it is suggested that the reader review [7].

In $\S 2$ the problem under consideration is defined in detail; certain auxiliary functions and other preliminary matters are considered in the next two sections. The pointwise bound is obtained in $\S 5$, while the generalization to the nonlinear case is considered in the following section. The last section contains some additional remarks. 
The techniques presented herein are based to a great extent on the results of the report [1] upon which this paper is based.

2. Mixed parabolic problem. Let $V(\eta)$ be an open connected $(N+1)$ dimensional domain of real variables $(x, y)=\left(x^{1}, x^{2}, \cdots, x^{N}, y\right)$ bounded by two hyperplanes $y=0$ and $y=\eta>0$ and an open surface $S(\eta)$ lying between these two planes. For fixed $y$ let

$$
D(y)=\{(x, y) \mid 0 \leqq y \leqq \eta,(x, y) \in \bar{V}(\eta)-\bar{S}(\eta)\}
$$

where $\bar{V}(\eta)$ and $\bar{S}(\eta)$ are the closures of $V(\eta)$ and $S(\eta)$ respectively.

The goal is to determine a pointwise bound at an arbitrary interior point $p=\left(x_{0}, y_{0}\right) \in D\left(y_{0}\right), 0<y_{0}<\eta$, of the boundary value problem:

$$
\left\{\begin{aligned}
J(W) & =\left(a^{i j} W_{, i}\right)_{, j}-z \frac{\partial W}{\partial y}=f(x, y) & & (x, y) \in V(\eta) \\
W(x, 0) & =g(x) & & (x, y) \in D(0) \\
\frac{\partial W}{\partial \nu} & =a^{i j} W_{, i} n_{j}=l(x, y) & & (x, y) \in S(\eta)
\end{aligned}\right.
$$

where the boundary data $f, g$ and $l$ are integrable and square integrable over their domains of definition and a sufficiently differentiable solution function $W=W(x, y)$ satisfying (2.2) is assumed to exist. As usual, the symbol ${ }_{i}$ indicates partial differentiation with respect to $x^{i}$ and the summation convention is used throughout.

The coefficients of (2.2) must satisfy certain auxiliary conditions. The components of the symmetric matrix $a^{i j}=a^{i j}(x, y)$ and the function $z=z(x, y)$ are continuous in $\bar{V}(\eta), a^{i j}$ is piecewise continuously differentiable with respect to the $x^{i}$ on every $D(y), 0<y<\eta$, and $z$ is piecewise continuously differentiable in $y$ along every line in $V(\eta)$ perpendicular to the $y$ hyperplane. To eliminate degeneracy, the existence of the following positive constants is assumed:

$$
\begin{gathered}
\min _{s\left(y_{0}\right)} \sum_{i=1}^{N} n_{i}^{2}=m_{1}>0 \\
\min _{V\left(y_{0}\right)} z(x, y)=m_{2}>0
\end{gathered}
$$

and for all $(x, y) \in \bar{V}\left(y_{0}\right)$

$$
0 \leqq a_{0} \sum_{1}^{N} \xi_{i}^{2} \leqq a^{i j} \xi_{i} \xi_{j} \leqq a_{1} \sum_{1}^{N} \xi_{i}^{2}
$$

where the $n_{i}$ are the first $N$ components of the unit outer normal $\left(n_{i}, n_{y}\right)=\left(n_{1}, \cdots, n_{N}, n_{y}\right)$, the $\xi_{i}$ are any $N$ numbers, and equality holds on the left if and only if all the $\xi_{i}$ are zero. At the point $p \in D\left(y_{0}\right)$, at which the pointwise bound is desired, it is necessary to 
require that $a^{i j}$ and $z$ satisfy a Lipschitz condition for $(x, y) \in \bar{V}\left(y_{0}\right)$, i.e. there exist numbers $A^{i j}$ and $A$ such that

$$
\left|a^{i j}(x, y)-a^{i j}(p)\right|\left\{\left(y_{0}-y\right)^{2}+\sum_{1}^{N}\left(x^{k}-x_{0}^{k}\right)^{2}\right\}^{-1 / 2} \leqq A^{i j}
$$

and

$$
|z(x, y)-z(p)|\left\{\left(y_{0}-y\right)^{2}+\sum_{1}^{N}\left(x^{k}-x_{0}^{k}\right)^{2}\right\}^{-1 / 2} \leqq A
$$

for every $(x, y) \in \bar{V}\left(y_{0}\right)$.

3. The parametrix. The parametrix function $\gamma_{p}$ is defined with respect to the point $p$ by

$$
\gamma_{p}=\left[4 \pi\left(y_{0}-y\right)\right]^{-N / 2} z(p)^{(N-2) / 2} \sqrt{a(p)} \exp \left[-z(p) \rho^{2}\left\langle 4\left(y_{0}-y\right)\right\rangle^{-1}\right]
$$

where

$$
\rho^{2}=a_{r s}(p)\left(x^{r}-x_{0}^{r}\right)\left(x^{s}-x_{0}^{s}\right)
$$

and $a(p)$ is the determinent of the inverse matrix $a_{i j}(p)$. On the surface $D\left(y_{0}\right)$, the parametrix and its derivatives are of course defined as the limits of these functions as $(x, y) \rightarrow\left(x, y_{0}\right)$ with $(x, y) \in V\left(y_{0}\right)$.

For $(x, y) \in V\left(y_{0}\right)$ the adjoint $\bar{J}(\psi)$ of $J(\psi)$ is given by

$$
\bar{J}(\psi)=\left(a^{i j} \psi,{ }_{, j}+\frac{\partial}{\partial y}(z \psi)\right.
$$

Lemma 3.1. The parametrix $\gamma_{p}$ possesses the following properties:

(a) At all points $(x, y) \in \bar{V}\left(y_{0}\right)-\{p\}$, the parametrix is twice continuously differentiable.

(b) The function

$$
\left(y_{0}-y\right)^{(N / 2)+\lambda} \exp \left[\zeta\left(y_{0}-y\right)^{-1} z(p) \rho^{2}\right] \bar{J}\left(\gamma_{p}\right)^{2}
$$

is integrable over $V\left(y_{0}\right)$ for $\zeta$ and $\lambda$ satisfying

$$
\zeta<\frac{1}{2} \text { and } \lambda>0 \text {. }
$$

(c) For any bounded function $\psi$ which is continuous on $\bar{V}\left(y_{0}\right)$, the parametrix is such that

$$
\lim _{\substack{y \rightarrow y_{0} \\ y<y_{0}}} \iint_{D(y)} \psi z \gamma_{p} d S=\psi(p)=\psi\left(x_{0}, y_{0}\right)
$$

Proof. From the definition of $\gamma_{p}$, it is obvious that part (a) is 
satisfied. To establish parts (b) and (c), perform the indicated integrations. The necessity for the constants given in (2.6) and (2.7) should be noted when part (b) is established.

4. An auxiliary function. In this section a sufficiently smooth function $\mathscr{F}_{p}$ is defined which is such that

$$
\mathscr{F}_{p} \geqq 0 \quad(x, y) \in \bar{V}\left(y_{0}\right)
$$

and

$$
\begin{aligned}
& \left\{\begin{array}{l}
\bar{J}\left(\mathscr{F}_{p}\right) \leqq 0 \\
\bar{J}\left(\mathscr{F}_{p}\right)<0
\end{array}\right. \\
& (x, y) \in \bar{V}\left(y_{0}\right) \\
& (x, y) \in \bar{V}\left(y_{0}-\varepsilon\right) \quad \forall \varepsilon>0
\end{aligned}
$$

where $\bar{J}$ is the adjoint of $J$. In addition it is necessary that $\mathscr{F}_{p}$ possess a singularity at $p \in D\left(y_{0}\right)$ which is of a slightly lower order than that of $\gamma_{p}$. The desired construction is accomplished by first defining

$$
\overline{\mathscr{F}_{p}}=\left(y_{0}-y\right)^{-(N-1) / 2} \exp \left[-(2 N-1) z(p) \rho^{2}\left\langle 8 N\left(y_{0}-y\right)\right\rangle^{-1}\right]
$$

and using (2.6) and (2.7) to observe that there exists positive numbers $\delta_{01}$ and $\rho_{0}$ which are such that

$$
\bar{J}\left(\overline{\mathscr{F}_{p}}\right) \leqq 0 \quad(x, y) \in\left\{x, y \mid \rho \leqq \rho_{0}, 0 \leqq y_{0}-y \leqq \delta_{01}\right\}
$$

and

$$
\bar{J}\left(\overline{\mathscr{F}_{p}}\right)<0 \quad(x, y) \in\left\{x, y \mid \rho \leqq \rho_{0}, \varepsilon \leqq y_{0}-y \leqq \delta_{01}\right\} \quad \forall \varepsilon>0 .
$$

Next $\delta_{02}$ is determined such that

$$
\frac{\partial z}{\partial y}\left(y_{0}-y\right)^{2}+\left(\frac{N-1}{2}\right)\left(y_{0}-y\right) z-\frac{(2 N-1) z(p) \rho_{0}^{2}}{8 N} z<0
$$

for all $(x, y) \in\left\{x, y \mid(x, y) \in \bar{V}\left(y_{0}\right)-\bar{V}\left(y_{0}-\delta_{02}\right)\right\}$. The number $\delta_{02}$ exists due to (2.4). Next set

$$
\delta_{0}=\min \left\{\delta_{01}, \delta_{02}\right\}
$$

and then define the desired auxiliary in terms of still undefined constants $\alpha$ and $\beta$.

$$
\begin{aligned}
& \mathscr{F}_{p}\left\{\begin{array}{r}
\overline{\mathscr{F}_{p}}+\alpha\left(y_{0}-y\right)^{-(N-1) / 2} \exp \left\{-(2 N-1) z(p) \rho_{0}^{2}\left\langle 8 N\left(y_{0}-y\right)\right\rangle^{-1}\right\} \\
(x, y) \in \bar{V}\left(y_{0}\right)-\bar{V}\left(y_{0}-\delta_{0}\right)
\end{array}\right. \\
& =\delta_{0}^{-(N-1+2 \beta) / 2}\left(y_{0}-y\right)^{\beta}\left[\exp \left\{-(2 N-1) z(p) \rho^{2}\left\langle 8 N\left(y_{0}-y\right)\right\rangle^{-1}\right\}\right. \\
& \left.+\alpha \exp \left\{-(2 N-1) z(p) \rho^{2}\left\langle 8 N\left(y_{0}-y\right)\right\rangle^{-1}\right\}\right] \quad(x, y) \in \bar{V}\left(y_{0}-\delta_{0}\right) \text {. }
\end{aligned}
$$


On $D\left(y_{0}\right), \mathscr{F}_{p}$ and its derivatives are defined as the limits of these functions as $(x, y) \rightarrow\left(x, y_{0}\right)$ with $(x, y) \in V\left(y_{0}\right)$. By inspection, (4.1) is valid for any positive $\alpha$. Using (4.6) and the definition of $\mathscr{F}_{p}$, it is easy to see that $\alpha>0$ may be chosen so that (4.2) is valid in the region $\left\{\bar{V}\left(y_{0}\right)-\bar{V}\left(y_{0}-\delta_{0}\right)\right\}$; and, with $\alpha$ fixed, we next use (2.4) to choose $\beta$ so that (4.2) is also valid for the region $\bar{V}\left(y_{0}-\delta_{0}\right)$.

As for differentiability in $\bar{V}\left(y_{0}\right)-\{p\}$, it follows from (4.8) that $\mathscr{F}_{p}$ and its first two partials with respect to the $x^{i}$ are continuous while the partial of $\mathscr{F}_{p}$ with respect to $y$ is only discontinuous across the surface $D\left(y_{0}-\delta_{0}\right)$.

Concerning the singularity of $\mathscr{F}_{p}$, it is easy to verify by means of Lemma 3.1 that the integral

$$
-\iiint_{V\left(y_{0}\right)}\left[\bar{J}\left(\mathscr{F}_{p}\right)\right]^{-1}\left[\bar{J}\left(\gamma_{p}\right)\right]^{2} d V
$$

exists and that for any function $\psi$ bounded in $\bar{V}\left(y_{0}\right)$

$$
\lim _{\substack{y \rightarrow y_{0} \\ y<y_{0}}} \iint_{D(y)} \psi \mathscr{F}_{p} d S=0
$$

5. Pointwise bound for the linear problem. The desired bound for the solution is obtained by choosing an arbitrary function $\varphi(x, y)$ which is such that $J(\varphi), \partial \varphi / \partial \nu$ and $\varphi(x, 0)$ approximate $f, l$, and $g$ respectively where $\varphi$ is required to be twice piecewise continuously differentiable with respect to the $x^{i}$ and piecewise continuously differentiable in $y$ for $(x, y) \in \bar{V}\left(y_{0}\right)$. Let

$$
\psi(x, y)=W(x, y)-\varphi(x, y)
$$

and compute

$$
\left\{\begin{aligned}
J(\psi) & =F(x, y) & & (x, y) \in V\left(y_{0}\right) \\
\psi(x, 0) & =G(x) & & (x, y) \in D(0) \\
\frac{\partial \psi}{\partial \nu} & =L(x, y) & & (x, y) \in S\left(y_{0}\right)
\end{aligned}\right.
$$

With $\bar{J}$ and $\gamma_{p}$ defined by (3.3) and (3.1) respectively, it follows from the divergence theorem with $0<y<y_{0}$

$$
\begin{array}{r}
\iiint_{V(y)}\left\{\gamma_{p} J(\psi)-\psi \bar{J}\left(\gamma_{p}\right)\right\} d V=\iint_{S(y)}\left\{\gamma_{p} \frac{\partial \psi}{\partial \nu}-\psi \frac{\partial \gamma_{p}}{\partial \nu}\right\} d S \\
-\iint_{D(y)} \gamma_{p} z \psi d S-\iint_{S(y)} n_{y} \gamma_{p} z \psi d S+\iint_{D(0)} \gamma_{p} z \psi d S .
\end{array}
$$

In (5.3), take the limit as $y \rightarrow y_{0}$, rearrange terms and apply Schwarz's 
inequality to obtain

$$
\begin{aligned}
& |W(p)-\chi(p)| \leqq\left|\iiint_{V\left(y_{0}\right)} \psi \bar{J}\left(\gamma_{p}\right) d V\right| \\
& \quad+\left\{\iint_{S\left(y_{0}\right)} \psi^{2} d S\right\}^{1 / 2}\left\{\iint_{S\left(y_{0}\right)}\left(\frac{\partial \gamma_{p}}{\partial \nu}+n_{y} z \gamma_{p}\right)^{2} d S\right\}^{1 / 2}
\end{aligned}
$$

where

$$
\begin{aligned}
\chi(p) & =\varphi\left(x_{0}, y_{0}\right)+\iint_{S\left(y_{0}\right)} L(x, y) \gamma_{p} d S \\
& +\iint_{D^{\prime}(0)} \gamma_{p} z G(x) d S-\iiint_{V\left(y_{0}\right)} \gamma_{p} F(x, y) d V .
\end{aligned}
$$

The terms on the right of (5.5) are all known and thus it only remains to bound the unknown terms on the right of (5.4).

First the surface integral is considered. To obtain the desired result, introduce the function $\tilde{\psi}$ given by

$$
\psi=\tilde{\psi} \exp \left[-K\left(y_{0}-y\right)\right]
$$

and calculate

$$
J(\psi)=\widetilde{J}(\widetilde{\psi}) \exp \left[-K\left(y_{0}-y\right)\right]
$$

where

$$
\widetilde{J}(\tilde{\psi})=J(\tilde{\psi})-K z \tilde{\psi}
$$

with $K$ a constant which is to be selected later. Next a bounded set of functions $f^{1}(x, y), \cdots, f^{N}(x, y)$ is chosen which possess bounded piecewise continuous partial derivatives with respect to the $x^{i}$ for $(x, y) \in \bar{V}\left(y_{0}\right)$ and which are such that

$$
\min _{S\left(y_{0}\right)} n_{i} f^{i}=m_{3}>0 \text {. }
$$

For starshaped domains such a set of functions was considered by Payne and Weinberger in $\S 3$ of [8]. From this reference it is clear that, if $S$ is starshaped in $x$ with respect to some line perpendicular to the $y$ hyperplane, then this line can be chosen as the $x$ origin with $f^{k}=x^{k}$. Also if $V\left(y_{0}\right)$ is a domain bounded by the $y=0$ and $y=y_{0}$ hyperplanes and by two surfaces each of which is starshaped in $x$ with respect to some line perpendicular to the $y$ hyperplane and if a cylindrical shell of Euclidean radius $r_{0}$ from this line can be imbedded in $V\left(y_{0}\right)$ such that its bases lie in $D(0)$ and $D\left(y_{0}\right)$, then the desired result is obtained by setting $f^{k}=x^{k}\left(r-r_{0}\right)$ where $r$ is the Euclidean distance of the surface $S\left(y_{0}\right)$ to the reference line. Additional infor- 
mation with respect to construction of the vector field $f^{k}$ may be obtained by referring to [3] or [4]. By extending these discussions to the parabolic case, it is evident that the functions $f^{k}$ may be defined for quite general domains.

To shorten the notation, the following convention will be employed in the remaining portion of the paper. When $y=y_{0}$ the symbols $S$ and $V$ replace $S\left(y_{0}\right)$ and $V\left(y_{0}\right)$.

With the $f^{i}$ defined as indicated above

$$
\begin{aligned}
\iint_{S} \widetilde{\psi} d S & \leqq \frac{1}{m_{3}} \iint_{S} \widetilde{\psi}^{2} f^{i} n_{i} d S \\
& \leqq \frac{\alpha_{1} M_{1}}{m_{3} a_{0}} \iiint_{V} a^{i j} \widetilde{\psi}_{, i} \widetilde{\psi}_{, j} d V+\left(\frac{1}{\alpha_{1} m_{3}}+\frac{M_{2}}{m_{3}}\right) \iiint_{V} \widetilde{\psi}^{2} d V
\end{aligned}
$$

where $\alpha_{1}$ is any positive number and

$$
M_{1}=\max _{V}\left\{\sum_{1}^{N} f^{i} f^{i}\right\}
$$

and

$$
M_{2}=\max _{V}\left\{f_{, i}^{i}\right\} .
$$

For the function $\tilde{\psi}$, it is clear that

$$
\begin{aligned}
& \iiint_{V} \widetilde{\psi} \bar{J}(\widetilde{\psi}) d V=\iint_{S}\left[\widetilde{\psi} \frac{\partial \widetilde{\psi}}{\partial \nu}-\frac{1}{2} n_{y} z \widetilde{\psi}^{2}\right] d S \\
& \quad+\iiint_{V}\left[-\widetilde{\psi},_{i} a^{i j} \widetilde{\psi},,_{j}-K z \widetilde{\psi}^{2}+\frac{1}{2}\left(\frac{\partial z}{\partial y}\right) \widetilde{\psi}^{2}\right] d V \\
& \quad-\iint_{D\left(y_{0}\right)} \widetilde{\psi}^{2} z d S+\iint_{D^{\prime}(0)} e^{2 K y_{0}} G^{2} z d S .
\end{aligned}
$$

By rearranging (5.13) and separating the product terms as indicated, it is easy to obtain

$$
\begin{aligned}
& \iiint_{V}\left[a^{i j} \widetilde{\psi}_{, i} \widetilde{\psi}_{, j}+\left(K m_{2}-\frac{1}{2} M_{3}-\frac{\alpha_{2}}{2}\right) \tilde{\psi}^{2}\right] d V+\frac{1}{2} \iint_{D\left(y_{0}\right)} \widetilde{\psi}^{2} z d S \\
& \leqq \frac{1}{2}\left(\alpha_{3}+M_{4}\right) \iint_{S} \widetilde{\psi}^{2} d S+\frac{1}{2} \iint_{D(0)} G^{2} z \exp \left[2 K y_{0}\right] d S \\
& +\frac{1}{2 \alpha_{2}} \iiint_{V} F^{2} \exp \left[2 K\left(y_{0}-y\right)\right] d V \\
& +\frac{1}{2 \alpha_{3}} \iint_{S} L^{2} \exp \left[2 K\left(y_{0}-y\right)\right] d S
\end{aligned}
$$

where 


$$
M_{3}=\max _{V}\left\{\frac{\partial z}{\partial y}\right\}, \quad M_{4}=\max _{s}\left\{-n_{y} z\right\}
$$

and the $\alpha$ 's are any positive numbers. Except for the first integral, the right side of (5.14) is completely know. In addition the left side has the same form as the right side of (5.10) except for the third term which may be neglected since $z$ satisfies (2.4). Inspection of (5.14) and (5.10) reveal that a bound for the integral of the square of $\tilde{\psi}$ over $S$ is available provided the arbitrary numbers $\alpha_{i}$ and $K$ are properly chosen. In a particular problem the best choice of these numbers would depend on the functions $F, G$, and $L$. For definiteness the following selection is made. Let

$$
\left\{\begin{array}{l}
\alpha_{1}=\frac{m_{3} a_{0}}{M_{1}}\left(1+M_{4}\right)^{-1} \\
\alpha_{2}=\alpha_{3}=1 \\
K=\frac{1}{m_{2}}\left\{\frac{\left(1+M_{4}\right)^{2} M_{1}}{m_{3}^{2} a_{0}}+\frac{\left(1+M_{4}\right) M_{2}}{m_{3}}+\frac{1}{2} M_{3}+\frac{1}{2}\right\}=K_{1}
\end{array}\right.
$$

Substitution of (5.16) into (5.10) and (5.14) yields in terms of the function $\psi$ :

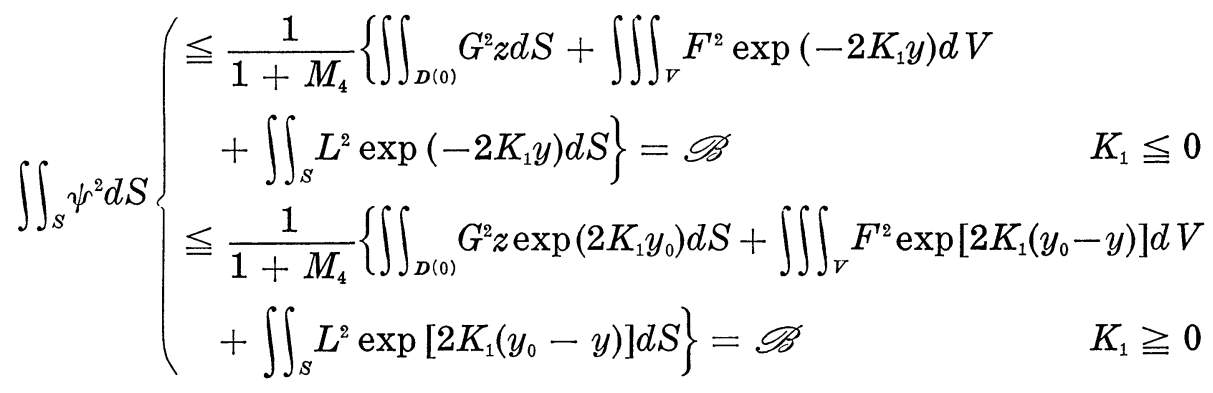

and thus a bound for the surface integral of (5.4) is available.

If a function $\Gamma_{p}$ can be determined which satisfies Lemma 3.1 and is such that

$$
\bar{J}\left(\Gamma_{p}\right)=0 \quad(x, y) \in \bar{V}\left(y_{0}\right)
$$

then it is clear that $\Gamma_{p}$ is a fundamental solution of $J$ and the volume integral on the right of (5.4) is zero. Dressel [6] and [7] established the existence of such a solution when the coefficients of the differential operator are sufficiently smooth. From these references it is clear that even when its existence is guaranteed such a function may be quite difficult to construct; hence in the following it is assumed that $\Gamma_{p}$ is 
not known and that the first term on the right of (5.4) must be bounded.

By means of the divergence theorem and the previous remarks of this section

$$
\begin{aligned}
& \iiint_{V}\left\{2 \mathscr{F}_{p} \psi J(\psi)-\psi^{2} \bar{J}\left(\mathscr{F}_{p}\right)+2 \mathscr{F}_{p} a^{i j} \psi,{ }_{i} \psi, j\right\} \\
& \quad \leqq \iint_{S}\left(\mathscr{F}_{p} L\right)^{2} d S+M_{5} \mathscr{B}+\iint_{D(0)} \mathscr{F}_{p} G^{2} z d S=\mathscr{J}
\end{aligned}
$$

where $\mathscr{B}$ is given by $(5.17)$ and

$$
M_{5}=\max _{s}\left\{0,1-\frac{\partial \mathscr{F}_{p}}{\partial \nu}-n_{y} \mathscr{F}_{p} z\right\}
$$

Due to the singularity of $\mathscr{F}_{p}$ each integral on the right of the inequality below exists

$$
\begin{aligned}
& -\left|\iiint_{V} \mathscr{F}_{p} \psi F d V\right| \\
& \quad \geqq-\left\{-\iiint_{V} \psi^{2} \bar{J}\left(\mathscr{F}_{p}\right) d V\right\}^{1 / 2}\left\{\iiint_{V}[-\bar{J}(\mathscr{F})]^{-1}\left(\mathscr{F}_{p} F\right)^{2} d V\right\}^{1 / 2},
\end{aligned}
$$

thus it is clear that

$$
\begin{aligned}
&\left\{-\iiint_{V} \bar{J}\left(\mathscr{F}_{p}\right) \psi^{2} d V\right\}^{1 / 2} \\
& \leqq\left\{\iiint_{V}\left[-\bar{J}\left(\mathscr{F}_{p}\right)\right]^{-1}\left(\mathscr{F}_{p} F\right)^{2} d V\right\}^{1 / 2} \\
&+\left\{\mathscr{J}+\iiint_{V}\left[-\bar{J}\left(F_{p}\right)\right]^{-1}\left(\mathscr{F}_{p} F\right)^{2} d V\right\}^{1 / 2} \\
&= \bar{J}^{1 / 2} .
\end{aligned}
$$

The desired bound is obtained by noting that

$$
\begin{aligned}
& \left|\iiint_{V} \psi \bar{J}\left(\gamma_{p}\right) d V\right| \\
& \quad \leqq\left\{\iiint_{V}\left[-\bar{J}\left(\mathscr{F}_{p}\right)\right]^{-1} \bar{J}\left(\gamma_{p}\right)^{2} d V\right\}^{1 / 2} \overline{\mathscr{J}}^{1 / 2}=\tilde{\mathscr{J}}
\end{aligned}
$$

where the existence of the integral on the right follows from part $b$ of Lemma 3.1.

By combining the results of this section, (5.4) may be rewritten as follows:

$$
|W(p)-\chi(p)| \leqq+\tilde{J}+\left\{\iint_{S}\left(\frac{\partial \gamma_{p}}{\partial \nu}+n_{y} z \gamma_{p}\right)^{2} d S\right\}^{1 / 2} \mathscr{B}{ }^{1 / 2}
$$


where the computable bounds $\mathscr{B}$ and $\tilde{\mathscr{J}}$ are given by (5.17) and (5.24) while the constant $\chi(p)$ may be obtained by reference to (5.5).

If problem (2.2) admits a solution $W$, then theoretically the pointwise bound may be made arbitrarily small by improvement of the approximation function $\varphi$. In this regard, note that the right side of (5.25) is zero if $F=L=G=0$. Furthermore, due to the form of the bound, it is clear that the result may be improved by means of the Rayleigh-Ritz technique. In order to ascertain the form of the bound obtained by the methods of this section, the above results are collected in the following theorem.

THEOREM 5.1. Pointwise bounds at an arbitrary interior point $p \in D\left(y_{0}\right)$ of the second initial-boundary value problem of parabolic type (5.2) are given by

$$
\begin{aligned}
\mid \psi(p) & -\iint_{S} \gamma_{p} L d S-\iint_{D(0)} \gamma_{p} z G d S \\
+ & \iiint_{V} \gamma_{p} F d V \mid \leqq k_{0}\left\{\iiint_{V} f_{0}(x, y) F^{2} d V\right\}^{1 / 2} \\
+ & \sum_{i=1}^{2} k_{i}\left\{\iint_{S} f_{i 1}(x, y) L^{2} d S+\iint_{D(0)} f_{i 2}(x) G^{2} d S\right. \\
+ & \left.\iiint \int_{V} f_{i 3}(x, y) F^{2} d V\right\}^{1 / 2}
\end{aligned}
$$

where $k_{i}, i=0,1,2$, are known constants and $f_{0}, f_{i j}, i=1,2$ and $j=$ $1,2,3$, are explicitly determined scaling functions. The constants $k_{i}$ and the functions $f_{0}$ and $f_{i j}$ are continuous with respect to the point $p \in D\left(y_{0}\right)$; however certain of the $k_{i}, f_{0}$ and $f_{i j}$ become arbitrarily large as $p$ approaches the boundary $S$.

6. Pointwise bound for the nonlinear problem. The mildly nonlinear problem for which an a priori bound is desired is as follows:

$$
\left\{\begin{aligned}
J(W) & =f(x, y, W, \nabla W) & & (x, y) \in V(\eta) \\
W(x, 0) & =g(x) & & (x, y) \in D(0) \\
\frac{\partial W}{\partial \nu} & =l(x, y, W) & & (x, y) \in S(\eta)
\end{aligned}\right.
$$

where the boundary data $f, g$, and $l$ are assumed to be square integrable over their domains of definition for any bounded continuous function $W$ which is continuously differentiable in $x$ for $(x, y) \in V(\eta)$. In addition $f$ and $l$ are required to satisfy a Lipschitz condition in all but their first two arguments. Hence there exist positive numbers $\widetilde{M}_{1}, \widetilde{M}_{2}$, $\tilde{M}^{i}, i=1, \cdots, N$ such that 


$$
\left\{\begin{array}{c}
\left|f\left(x, y, W_{1}, \nabla W_{1}\right)-f\left(x, y, W_{2}, \nabla W_{2}\right)\right| \leqq \tilde{M}_{1}\left|W_{1}-W_{2}\right| \\
+\tilde{M}^{i}\left|W_{1, i}-W_{2, i}\right| \\
\mid x, y) \in V(\eta) ;-\infty<W, \nabla W<\infty \\
\left|l\left(x, y, W_{1}\right)-l\left(x, y, W_{2}\right)\right| \leqq \widetilde{M}_{2}\left|W_{1}-W_{2}\right| \\
(x, y) \in S(\eta) ;-\infty<W<\infty .
\end{array}\right.
$$

The present problem is a generalization of the problem of the last section; thus, except as altered by this section, all conditions of $\S 2$ remain in effect. Specifically, the differentiability requirements for the coefficients, the Lipschitz condition which the $a^{i j}$ are to satisfy at $p \in D\left(y_{0}\right)$, as well as the condition for the outer normal on $S\left(y_{0}\right)$ are all as stated in $\S 2$ of the paper.

The pointwise bound is obtained in a manner very analogous to that of $\S 5$ and hence frequent use will be made of the applicable equations and inequalities of the linear case.

A bound at an arbitrary interior point $p \in D\left(y_{0}\right)$ is obtained by choosing a function $\varphi(x, y)$ which is such that $J(\varphi) ; \partial \varphi / \partial \nu$ and $\varphi(x, 0)$ approximate $f(x, y, \varphi, \nabla \varphi), l(x, y, \varphi)$ and $g(x)$ respectively. The function $\varphi(x, y)$ given by (5.1) is introduced and we calculate.

$$
\begin{cases}J(\psi)=f(x, y, W, \nabla W)-f(x, y, \varphi, \nabla \varphi)+F(x, y) & (x, y) \in V\left(y_{0}\right) \\ \psi(x, 0)=G(x) & (x, y) \in D(0) \\ \frac{\partial \psi}{\partial \nu}=l(x, y, W)-l(x, y, \varphi)+L(x, y) & (x, y) \in S\left(y_{0}\right)\end{cases}
$$

where

$$
\begin{aligned}
& F(x, y)=f(x, y, \varphi, \nabla \varphi)-J(\varphi) \\
& L(x, y)=l(x, y, \varphi)-\frac{\partial \varphi}{\partial \nu} .
\end{aligned}
$$

Equation (5.4) becomes

$$
\begin{aligned}
& |W(p)-\chi(p)| \leqq\left|\iiint_{V\left(y_{0}\right)} \psi \bar{J}\left(\gamma_{p}\right) d V\right| \\
& +\left|\iiint_{V\left(y_{0}\right)} \gamma_{p}\{f(x, y, W, \nabla W)-f(x, y, \varphi, \nabla \varphi)\} d V\right| \\
& +\left\{\iint_{S\left(y_{0}\right)} \psi^{2} d S\right\}^{1 / 2}\left\langle\left\{\iint_{S\left(y_{0}\right)}\left(\frac{\partial \gamma_{p}}{\partial \nu}+n_{y} z \gamma_{p}\right)^{2} d S\right\}^{1 / 2}\right. \\
& \left.\quad+\tilde{M}_{2}\left\{\iint_{S} \gamma_{p}^{2} d S\right\}^{1 / 2}\right\rangle
\end{aligned}
$$

where $\chi(p)$ is given by (5.5). As in $\S 5$, the first step is to obtain an a priori estimate for the unknown boundary integral. By using techniques similar to those employed for the linear case, it is easy to establish the following generalization of (5.14) 


$$
\begin{aligned}
& \iiint_{V}\left[\left(1-\frac{M_{6} \alpha_{4}}{2 a_{0}}\right) a^{i j} \widetilde{\psi}_{, i} \widetilde{\psi}_{, j}+\left(K m_{2}-\frac{M_{3}}{2}-\frac{\alpha_{2}}{2}-\widetilde{M}_{1}-\frac{1}{2 \alpha_{4}}\right) \widetilde{\psi}^{2}\right] d V \\
& \left.\quad+\frac{1}{2} \iint_{D\left(y_{0}\right)} \widetilde{\psi}^{2} z d S \leqq \frac{1}{2}\left(\alpha_{3}+M_{4}+2 \widetilde{M}_{2}\right)\right] \int_{S} \widetilde{\psi}^{2} d S \\
& \quad+\frac{1}{2} \iint_{D(0)} G^{2} z \exp \left(2 K y_{0}\right) d S+\frac{1}{2 \alpha_{2}} \iiint_{V} F^{2} \exp \left[2 K\left(y_{0}-y\right)\right] d V \\
& \quad+\frac{1}{2 \alpha_{3}} \iint_{S} L^{2} \exp \left[2 K\left(y_{0}-y\right)\right] d S
\end{aligned}
$$

where

$$
M_{6}=\sum_{1}^{N}\left(\widetilde{M}^{i}\right)^{2}
$$

and the $\alpha$ 's are arbitrary positive numbers. While the best choice of the $\alpha_{i}$ and $K$ is a function of $F, G$ and $L$, for definiteness the following selection is made. Let

$$
\left\{\begin{aligned}
\alpha_{1}= & \frac{a_{0} m_{3}}{4 M_{1}}\left(\tilde{M}_{2}+\frac{1}{2}+\frac{1}{2} M_{4}\right)^{-1} \\
\alpha_{2}= & \alpha_{3}=1 \\
\alpha_{4}= & \frac{a_{0}}{M_{6}} \\
K= & \frac{1}{m_{2}}\left\{\frac{M_{3}}{2}+\tilde{M}_{1}+\frac{M_{6}}{2 a_{0}}+\frac{1}{2}\right. \\
& \left.+\left(2 \widetilde{M}_{2}+1+M_{4}\right)\left\langle\frac{2\left(2 \widetilde{M}_{2}+1+M_{4}\right) M_{1}}{a_{0} m_{3}^{2}}+\frac{M_{2}}{m_{3}}\right\rangle\right\}=K_{1} .
\end{aligned}\right.
$$

With the $\alpha_{i}$ and $K$ chosen by (6.8), we may combine (6.6) and (5.10) to obtain a result equivalent to $(5.17)$ except that the factor $\left(1+M_{4}\right)^{-1}$ on the left is replaced by $\left(2 \widetilde{M}_{2}+1+M_{4}\right)^{-1}$.

To bound the first term on the right of $(6.5)$, replace $(5.20)$ by

$$
\begin{gathered}
\iiint_{V}\left\{2 \mathscr{F}_{p} \widetilde{\psi} \widetilde{J}(\widetilde{\psi})-\widetilde{\psi}^{2} \bar{J}\left(\mathscr{F}_{p}\right)+2 \mathscr{F}_{p} \widetilde{\psi}_{, i} a^{i j} \widetilde{\psi}_{, j}+2 K z \mathscr{F}_{p} \widetilde{\psi}^{2}\right\} d V \\
\leqq \\
\quad \iint_{S}\left(\mathscr{F}_{p} L\right)^{2} \exp \left[2 K\left(y_{0}-y\right)\right] d S+M_{7} \mathscr{B} \exp \left(2 K y_{0}\right) \\
\quad+\iint_{D(0)} \mathscr{F}_{p} G^{2} z \exp \left(2 K y_{0}\right) d S=\mathscr{J}(K)
\end{gathered}
$$

where $\mathscr{J}(K)$ is a function of the arbitrary constant $K$ and

$$
M_{7}=\max _{s}\left\{0,1+2 \tilde{M}_{2} \mathscr{F}_{p}-\frac{\partial \mathscr{F}_{p}}{\partial \nu}-n_{y} \mathscr{F}_{p} z\right\} .
$$

Since $f$ satisfies a Lipschitz condition in all but its first two variables, 
it is clear that

$$
\begin{aligned}
& \iiint_{V} 2 \mathscr{F}_{p} \widetilde{\psi} \widetilde{J}(\widetilde{\psi}) d V \geqq 2 \iiint_{V} \mathscr{F}_{p} \widetilde{\psi} F \exp \left[K\left(y_{0}-y\right)\right] d V \\
& \quad-\left(2 \widetilde{M}_{1}+\alpha_{5}\right) \iiint_{V} \mathscr{F}_{p} \widetilde{\psi}^{2} d V-\frac{M_{6}}{\alpha_{5} a_{0}} \iiint_{V} \mathscr{F}_{p} a^{i j} \widetilde{\psi},_{i} \widetilde{\psi}_{, j} d V .
\end{aligned}
$$

Comparison of (6.9) and (6.11) leads to a selection of the arbitrary constants as follows:

$$
\left\{\begin{array}{l}
\alpha_{5}=\frac{M_{6}}{2 a_{0}} \\
K=\frac{1}{2 m_{2}}\left\{2 \widetilde{M}_{1}+\frac{M_{6}}{2 a_{0}}\right\}=K_{2} .
\end{array}\right.
$$

An inequality analogous to (5.22) may now be used to obtain

$$
\begin{aligned}
\left|\iiint_{V} \psi \bar{J}\left(\gamma_{p}\right) d V\right| \leqq\left\{\iiint_{V}\left[-\bar{J}\left(\mathscr{F}_{p}\right)\right]^{-1} \bar{J}\left(\gamma_{p}\right)^{2} d V\right\}^{1 / 2} \\
\quad \cdot\left\langle\left\{\iiint_{V}\left[-\bar{J}\left(\mathscr{F}_{p}\right)\right]^{-1}\left(\mathscr{F}_{p} F\right)^{2} \exp \left[2 K_{2}\left(y_{0}-y\right)\right] d V\right\}^{1 / 2}\right. \\
\left.+\left\{\mathscr{J}\left(K_{2}\right)+\iiint_{V}\left[-\bar{J}\left(\mathscr{F}_{p}\right)\right]^{-1}\left(\mathscr{F}_{p} F\right)^{2} \exp \left[2 K_{2}\left(y_{0}-y\right)\right] d V\right\}^{1 / 2}\right\rangle=\tilde{J}
\end{aligned}
$$

which is a result analogous to (5.24).

The second term on the right of (6.5) is bounded by using similar techniques; due to the Lipschitz condition, it is clear that

$$
\begin{aligned}
&\left|\iiint_{V} \gamma_{p}\{f(x, y, W, \nabla W)-f(x, y, \varphi, \nabla \varphi)\} d V\right| \\
& \leqq\left\{\iiint_{V} \gamma_{p}^{2} \mathscr{F}_{p}^{-1} \exp \left[2 K\left(y_{0}-y\right)\right] d V\right\}^{1 / 2} \\
& \cdot\left\{\iiint_{V}\left[2 \widetilde{M}_{1}^{2} \mathscr{F}_{p} \widetilde{\psi}^{2}+2 \frac{M_{6}}{a_{0}} \mathscr{F}_{p} \widetilde{\psi}_{, i} a^{i j} \widetilde{\psi}_{, j}\right] d V\right\}^{1 / 2} .
\end{aligned}
$$

The first integral on the right of the last inequality exists; to bound the second observe that

$$
\begin{aligned}
& \left|2 \mathscr{F}_{p} \tilde{\psi} F \exp \left[K\left(y_{0}-y\right)\right]\right| \\
& \quad \leqq \frac{1}{\alpha_{6}} \mathscr{F}_{p} F^{2} \exp \left[2 K\left(y_{0}-y\right)\right]+\alpha_{6} \mathscr{F}_{p} \widetilde{\psi}^{2}
\end{aligned}
$$

and then combine $(6.15),(6.11)$ and $(6.9)$ to obtain

$$
\begin{aligned}
\iiint_{V}\{ & -\frac{1}{\alpha_{8}} \mathscr{F}_{p} F^{2} \exp \left[2 K\left(y_{0}-y\right)\right]+\left(2 K z-\alpha_{6}-2 \widetilde{M}_{1}-\alpha_{5}\right) \mathscr{F}_{p} \widetilde{\psi}^{2} \\
& \left.+\left(2-\frac{M_{8}}{\alpha_{5} a_{0}}\right) \mathscr{F}_{p} a^{i j} \widetilde{\psi}_{, i} \widetilde{\psi}_{, j}\right\} d V \leqq \mathscr{J}(K) .
\end{aligned}
$$


The arbitrary constants may now be fixed so that

$$
\left\{\begin{array}{l}
\alpha_{5}=M_{6} a_{0}^{-1} \\
\alpha_{6}=1 \\
K=\frac{1}{2 m_{2}}\left\{1+2 \tilde{M}_{1}+M_{6} a_{0}^{-1}+a_{0} M_{6}^{-1} \tilde{M}_{1}^{2}\right\}=K_{3} .
\end{array}\right.
$$

After substitution of (6.17) into (6.16) the desired bound may be obtained by writing

$$
\left|\iiint_{V} \gamma_{p}\{f(x, y, W, \nabla W)-f(x, y, \varphi, \nabla \phi)\}\right|
$$

$$
\begin{aligned}
& \leqq\left\{\iint_{V} \gamma_{p}^{2} \mathscr{F}_{p}^{-1} \exp \left[2 K_{3}\left(y_{0}-y\right)\right] d V\right\}^{1 / 2} \\
& \cdot\left\langle\left(2 M_{6} a_{0}^{-1}\right)\left\{\mathscr{J}\left(K_{3}\right)+\iiint_{V} \mathscr{F}_{p} F^{2} \exp \left[2 K_{3}\left(y_{0}-y\right)\right] d V\right\}\right\rangle^{1 / 2}=\mathscr{K} .
\end{aligned}
$$

By means of the inequalities developed in this section, (6.5) is rewritten as:

$$
\begin{aligned}
& |W(p)-\chi(p)| \leqq \tilde{\mathscr{J}}+\mathscr{K}+\left\langle\left\{\widetilde{M}_{2} \iint_{S} \gamma_{p}^{2} d S\right\}^{1 / 2}\right. \\
& \left.\quad+\left\{\iint_{S}\left(\frac{\partial \gamma_{p}}{\partial \nu}+n_{y} z \gamma_{p}\right)^{2} d S\right\}^{1 / 2}\right\rangle \mathscr{B}^{1 / 2}
\end{aligned}
$$

which is the pointwise bound that was desired. The results of this section may be collected in the following theorem.

THEOREM 6.1. Pointwise bounds at an arbitrary interior point of the nonlinear second initial-boundary value problem of parabolic type (6.1) are given by

$$
\begin{aligned}
\mid \psi(p) & -\iint_{S} \gamma_{p} L d S-\iint_{D^{\prime}(0)} \gamma_{p} z G d S+\iiint_{V} \gamma_{p} F d V \mid \\
\leqq & k_{0}\left\{\iiint_{V} f_{0}(x, y) F^{2} d V\right\}^{1 / 2}+\sum_{i=1}^{3} k_{i}\left\{\iint_{S^{\prime}} f_{i 1}(x, y) L^{2} d S\right. \\
& \left.+\iint_{D^{\prime}(0)} f_{i 2}(x) G^{2} d S+\iiint_{V} f_{i 3}(x, y) F^{2} d V\right\}^{1 / 2}
\end{aligned}
$$

where $k_{i}, i=0,1,2,3$, are known constants and $f_{0}, f_{i j}, i=1,2,3$ and $j=1,2,3$, are explicitly determined scaling functions. The constants $k_{i}$ and the functions $f_{0}$ and $f_{i j}$ are continuous with respect to the point $p \in D\left(y_{0}\right)$; however, certain of the $k_{i}, f_{0}$ and $f_{i j}$ become arbitrarily large as $p$ approaches the boundary $S$.

7. Additional remarks. Since it was possible to handle the nonlinear case of $\S 6$, it should be noted that the form of the operator 
$J$ and the symmetry of the $a^{i j}$ may be relaxed. Thus the method may clearly be used to obtain bounds in either of the following cases:

$$
J(W)=\left(a^{i j} W_{, i}\right)_{, j}-z \frac{\partial W}{\partial y}
$$

or

$$
J(W)=a^{i j} W_{, i j}-z \frac{\partial W}{\partial y}
$$

where the matrix $a^{i j}$ is not symmetric. It is evident that the methods of this paper can be easily adapted to apply to systems of parabolic equations or to higher order operators; as of this writing, the author has not carried out such generalizations in detail. Specific results have, however, been obtained for nonlinear parabolic problem of the Dirichlet type and for certain degenerate parabolic problems. These results will appear in sequels which are soon to appear.

The author is indebted to Professor L. C. Payne for valuable advice and suggestions.

\section{REFERENCES}

1. F. Bellar, Pointwise bounds in parabolic and elliptic partial differential equations, Tech. Note BN-246, Inst. Fluid Dynamics, University of Maryland, College Park, 1961.

2. J. Bramble and L. Payne, Bounds in the Neumann problem for second order uniformly elliptic operators, Pacific J. Math. 12 (1962), 823-833.

3. - Bounds for solutions of second order elliptic partial differential equations, Cont. to Diff. Eqns. 1 (1963), 95-127.

4. - Inequalities for solutions of mixed boundary value problems for elastic plates, J. Research Natl. Bur. Standards, 68B (1964), 75-91.

5. F. Dressel, The fundamental solntion of the parabolic equation, Duke Math. J. 7 (1940), 186-203.

6. The fundamental solution of the parabolic equation, Duke Math. J. 13 (1946), 61-70.

7. A. Friedman, Partial differential equations of parabolic type, Prentice-Hall, Englewood Cliffs, 1964.

8. L. Payne and H. Weinberger, New bounds for solutions of second order elliptic partial differential equations, Pacific J. Math. 8 (1958), 551-573.

Received June 18, 1965, and in revised form October 18, 1965. This paper is part of a thesis submitted to the faculty of the University of Maryland in partial fulfillment of the requirements for the degree of Doctor of Philosophy. The research upon which this paper is based was completed while the author attended the University of Maryland under the sponsorship of the U. S. Naval Postgraduate School.

Office of Naval Research, Washington, D. C. 



\title{
PACIFIC JOURNAL OF MATHEMATICS
}

\author{
EDITORS
}

\section{H. SAMELSON}

Stanford University

Stanford, California

J. P. JANS

University of Washington

Seattle, Washington 98105
J. DugundJI

University of Southern California

Los Angeles, California 90007

RICHARD ARENS

University of California

Los Angeles, California 90024

\section{ASSOCIATE EDITORS}

\section{E. F. BECKENBACH}

B. H. NEUMANN

F. WOLF

K. YosidA

\section{SUPPORTING INSTITUTIONS}

\author{
UNIVERSITY OF BRITISH COLUMBIA \\ CALIFORNIA INSTITUTE OF TECHNOLOGY \\ UNIVERSITY OF CALIFORNIA \\ MONTANA STATE UNIVERSITY \\ UNIVERSITY OF NEVADA \\ NEW MEXICO STATE UNIVERSITY \\ OREGON STATE UNIVERSITY \\ UNIVERSITY OF OREGON \\ OSAKA UNIVERSITY \\ UNIVERSITY OF SOUTHERN CALIFORNIA
}

\author{
STANFORD UNIVERSITY \\ UNIVERSITY OF TOKYO \\ UNIVERSITY OF UTAH \\ WASHINGTON STATE UNIVERSITY \\ UNIVERSITY OF WASHINGTON \\ AMERICAN MATHEMATICAL SOCIETY \\ CHEVRON RESEARCH CORPORATION \\ TRW SYSTEMS \\ NAVAL ORDNANCE TEST STATION
}

Mathematical papers intended for publication in the Pacific Journal of Mathematics should be typewritten (double spaced). The first paragraph or two must be capable of being used separately as a synopsis of the entire paper. It should not contain references to the bibliography. Manuscripts may be sent to any one of the four editors. All other communications to the editors should be addressed to the managing editor, Richard Arens at the University of California, Los Angeles, California 90024.

50 reprints per author of each article are furnished free of charge; additional copies may be obtained at cost in multiples of 50 .

The Pacific Journal of Mathematics is published monthly. Effective with Volume 16 the price per volume (3 numbers) is $\$ 8.00$; single issues, $\$ 3.00$. Special price for current issues to individual faculty members of supporting institutions and to individual members of the American Mathematical Society: $\$ 4.00$ per volume; single issues $\$ 1.50$. Back numbers are available.

Subscriptions, orders for back numbers, and changes of address should be sent to Pacific Journal of Mathematics, 103 Highland Boulevard, Berkeley 8, California.

Printed at Kokusai Bunken Insatsusha (International Academic Printing Co., Ltd.), No. 6, 2-chome, Fujimi-cho, Chiyoda-ku, Tokyo, Japan.

PUBLISHED BY PACIFIC JOURNAL OF MATHEMATICS, A NON-PROFIT CORPORATION

The Supporting Institutions listed above contribute to the cost of publication of this Journal, but they are not owners or publishers and have no responsibility for its content or policies. 


\section{Pacific Journal of Mathematics \\ Vol. 19, No. $2 \quad$ June, 1966}

Leonard Daniel Baumert, Extreme copositive quadratic forms . ......... 197

Fred James Bellar, Jr., Pointwise bounds for the second initial-boundary value problem of parabolic type ........................ 205

L. Carlitz and David Paul Roselle, Restricted bipartite partitions ........ 221

Robin Ward Chaney, On the transformation of integrals in measure space ........................................... 229

Colin W. Clark, An embedding theorem for function spaces ........... 243

Edwin Duda, A theorem on one-to-one mappings................ 253

Ben Fitzpatrick, Jr. and Donald Reginald Traylor, Two theorems on metrizability of Moore spaces.......................... 259

Allen Roy Freedman, An inequality for the density of the sum of sets of vectors in $n$-dimensional space ....................... 265

Michael Friedberg, On representations of certain semigroups .......... 269

Robert William Gilmer, Jr., The pseudo-radical of a commutative ring . . . . 275

Hikosaburo Komatsu, Fractional powers of operators ............... 285

Daniel Rider, Transformations of Fourier coefficients ................. 347

David Alan Sánchez, Some existence theorems in the calculus of variations ...................................... 357

Howard Joseph Wilcox, Pseudocompact groups............... 365

William P. Ziemer, Some lower bounds for Lebesgue area ............. 381 